\title{
Generalized Consensus for Practical Fault Tolerance
}

\author{
Mohit Garg \\ Virginia Tech \\ mohitce@vt.edu \\ Balaji Arun \\ Virginia Tech \\ balajia@vt.edu
}

\author{
Sebastiano Peluso \\ Virginia Tech \\ peluso.sebastiano@gmail.com \\ Binoy Ravindran \\ Virginia Tech \\ binoy@vt.edu
}

\begin{abstract}
Despite extensive research on Byzantine Fault Tolerant (BFT) systems, overheads associated with such solutions preclude widespread adoption. Past efforts such as the Cross Fault Tolerance (XFT) model address this problem by making a weaker assumption that a majority of nodes are correct and communicate synchronously. Although XPaxos of Liu et al. (applying the XFT model) achieves similar performance as Paxos, it does not scale with the number of faults. Also, its reliance on a single leader introduces considerable downtime in case of failures. We present Elpis, the first multi-leader XFT consensus protocol. By adopting the Generalized Consensus specification, we were able to devise a multi-leader protocol that exploits the commutativity property inherent in the commands ordered by the system. Elpis maps accessed objects to non-faulty replicas during periods of synchrony. Subsequently, these replicas order all commands which access these objects. The experimental evaluation confirms the effectiveness of this approach: Elpis achieves up to $2 \mathrm{x}$ speedup over XPaxos and up to 3.5x speedup over state-ofthe-art Byzantine Fault-Tolerant Consensus Protocols.
\end{abstract}

CCS Concepts - Security and privacy $\rightarrow$ Distributed systems security; $\bullet$ Software and its engineering $\rightarrow$ Software fault tolerance;

Keywords Consensus; Generalized Consensus; Byzantine Fault Tolerance; Collision Recovery; Blockchain

\section{ACM Reference Format:}

Mohit Garg, Sebastiano Peluso, Balaji Arun, and Binoy Ravindran. 2019. Generalized Consensus for Practical Fault Tolerance. In Middleware '19: Middleware '19: 20th International Middleware Conference, December 8-13, 2019, Davis, CA, USA. ACM, New York, NY, USA, 13 pages. https://doi.org/10.1145/3361525.3361536

Permission to make digital or hard copies of all or part of this work for personal or classroom use is granted without fee provided that copies are not made or distributed for profit or commercial advantage and that copies bear this notice and the full citation on the first page. Copyrights for components of this work owned by others than ACM must be honored. Abstracting with credit is permitted. To copy otherwise, or republish, to post on servers or to redistribute to lists, requires prior specific permission and/or a fee. Request permissions from permissions@acm.org.

Middleware '19, December 8-13, 2019, Davis, CA, USA

() 2019 Association for Computing Machinery.

ACM ISBN 978-1-4503-7009-7/19/12.

https://doi.org/10.1145/3361525.3361536

\section{Introduction}

Consensus solutions underpin numerous distributed systems - from horizontally scalable databases $[2,13,16]$ and keyvalue stores [3] to distributed synchronization services [12, 17] - providing strong consistency, fault-tolerance, and high availability. These systems employ State Machine Replication [29] where Consensus algorithms are used to achieve agreement on a common order among concurrent client requests that each node in a distributed system should execute, even in the presence of faults.

Consensus algorithms are designed using two prominent fault models: the Crash Fault Tolerance (CFT) model and the Byzantine Fault Tolerance (BFT) model [23]. CFT protocols do not tolerate any non-crash faults - even accidental faults like hardware errors, misconfigurations, and software bugs, that are increasingly common in production systems today $[1,4,5]$. BFT protocols, on the other hand, shield applications from non-crash faults, including malicious actors, but are expensive, requiring more resources and complex messaging patterns. Notably, in geo-scale deployments where round-trip timings (RTT) are high, BFT protocols have significantly higher client-perceived latencies, discouraging widespread adoption. Various approaches $[8,18,25]$ improve the performance of BFT protocols, but the lower bounds of the BFT model [23] prevents from reducing both the number of communication steps as well as the quorum size, which is essential for reducing wide-area latencies.

Most practical systems today operate in secure networks with solutions in place to thwart malicious attacks like distributed denial-of-service [31]. For these systems, the Cross Fault Tolerance (XFT) model [24] achieves a favorable tradeoff between the CFT and BFT models. Mainly, the XFT model relaxes the assumption that the adversary can launch coordinated attacks, which is unlikely in geo-scale deployments but is sufficient to shield applications from crash faults, network faults, and non-crash non-malicious faults. This enables the XFT model to use the same quorum size and the same number of communication steps as the CFT model by assuming that a majority of processes are correct and synchronous.

XPaxos [24], the lone XFT protocol, is leader-based with performance similar to CFT-based Raft/Multi-Paxos [27] 
protocols. While the XFT model is built with an assumption that befits the geo-replicated setting, the accompanying algorithm, XPaxos, provides poor scalability and performance. XPaxos inherits the shortcomings of leader-based approaches: imbalanced load distribution, where the leader does more work than other nodes; high latency for requests originating from non-leader nodes due to the requirement of forwarding requests to the leader; and the inability to deliver any commands whenever the current leader is slow or Byzantine pending a leader/view-change.

To address these aspects, we present Elpis, the first multileader XFT consensus protocol that exploits the underlying commutativity of commands to provide fast decisions in three communication steps from any node, in the common case. We achieve this by exploiting workload locality that is very common in geo-scale deployments. The core idea of Elpis is enabling ownership at a finer granularity. Rather than having a single leader that is responsible for ordering all commands regardless of their commutative property, we assign ownership to individual nodes such that each node mostly proposes only commutative commands with respect to other nodes. As a result, each node is responsible for deciding the order of all commands that commute with other nodes. We define commutativity by the object(s) that a command accesses during its execution. With this, we assign ownership to nodes on a per-object basis. Such ownership assignment guarantees that no other node will propose conflicting commands, and thus, fast decisions in three communication steps can be achieved from the owner nodes. Furthermore, clients benefit from workload locality by sending requests to the closest node (with ownership) and observe optimal latencies.

Elpis also allows for dynamic ownership changes. Individual nodes can gain ownership of any object(s) using a special ownership acquisition phase. We recognize the conflicts between multiple nodes trying to acquire the ownership of the same object(s) concurrently. We address this using a rectification mechanism that follows the ownership acquisition phase during conflicts and minimizes the number of retries to acquire ownership. Additionally, while XPaxos and BFT protocols like PBFT [15] use a three-phase view-change/leader election sub-protocol in addition to the normal operation phases, Elpis requires just one additional phase (two for regular operation) for liveness. This linear procedure of Elpis also improves on the combinatorial view-change mechanism of XPaxos.

We implemented Elpis and competitors - $\mathrm{M}^{2}$ Paxos [28], XPaxos, PBFT, Zyzzyva [18] - in Java, using the JGroups messaging toolkit for the first two protocols and BFT-SMaRT [10] a highly optimized implementation of PBFT to implement Zyzzyva. We extensively evaluated each of the existing solutions and contrasted their performances to show the gains achieved by our solution. To summarize, Elpis achieves up to $2 \mathrm{x}$ speedup over XPaxos and up to $3.5 \mathrm{x}$ speedup over the state-of-the-art BFT Consensus Protocols.
The core contributions of this paper are:

1. The design and implementation of the first multi-leader Cross Fault Tolerant (XFT) consensus protocol

2. An ownership conflict resolution protocol that minimizes retries due to proposer contention using a more cohesive algorithm.

3. An extensive evaluation and comparison to the existing state-of-the-art in the BFT space.

The rest of the paper is organized as follows: In Section 2, we discuss the desirable properties which are seemingly amiss from production systems today. Section 3 presents the system model and assumptions. In Section 4.1, we introduce Elpis at a high level, while in Section 4.3, we delve into the details and present the algorithm pseudocode. Section 5 presents the correctness proof of Elpis supported by a TLA+ specification [20] for the algorithm. We evaluate our solution and competitors in Section 6, and we summarize the existing state-of-the-art solutions that are related to our contribution, Elpis in Section 7. We conclude in Section 8.

\section{Motivation}

One of the primary motivations for Elpis is to provide strong consistency in geo-replication. Under the CAP theorem [11], only one out of two - Consistency or Availability - can be guaranteed in the presence of a network partition. Distributed systems like Cassandra [14] and DynamoDB [30] choose availability over consistency under a network partition. While availability can not be assured in real-world systems, these systems hand-over the burden of ensuring consistency to application developers. Additionally, such systems cannot safeguard applications from faults such as data corruptions without additional mechanisms. In contrast, with Elpis, the objective is to favor strong consistency, while striving to provide high availability under faults. Specifically, using the localized ownership mechanism, we ensure that a faulty node does not bring the system to a standstill (pending leader election), unlike single-leader based protocols. As long as a majority of nodes are up, clients requests are executed.

Moreover, Elpis empowers shard-based systems to guarantee linearizability on multi-shard operations. Most geographically distributed systems run a per-shard consensus protocol (e.g., CockroachDB [2], Spanner [16]) to achieve scalability with the number of nodes. However, guaranteeing linearizability on multi-shard transactions is non-trivial and requires additional hardware components such as GPS clocks which timestamp transactions to establish order (Example, CockroachDB uses HLC (hybrid logical clocks), and YugaByte [6] uses Hybrid Time). Such systems can instead depend on a single instance of Elpis to guarantee linearizability as well as scalability without the need for additional mechanisms. Multi-shard operations can be easily executed without complicated cross-shard transactions by the application layer. 
Furthermore, Elpis provides an appealing trade-off between CFT and BFT protocols. While CFT protocols cannot tolerate non-crash faults, BFT protocols require more nodes and larger quorums to tolerate the same number of faults as CFT protocols. The need for bigger quorums in the BFT model is due to the assumption that $t$ non-faulty processes could be slow in responding in the presence of $t$ Byzantine processes. Therefore, an additional $t$ non-faulty processes are required to distinguish between messages from non-faulty and Byzantine processes. Hence, a quorum of size $2 t+1$ out of $3 t+1$ processes is required for consensus protocols that employ the BFT model. In practice, this assumption implies that an adversary can affect the network on a wide scale as well as attack multiple nodes all in a coordinated fashion. This is a strong assumption, especially for geo-replicated systems where data-centers are distributed around the world and linked using secure networks. The XFT model, instead, provides the same quorum size and uses the same number of communication steps as the CFT model.

Multi-leader consensus solutions [7, 26, 28] have been proposed for the CFT model in recent years to address the aforementioned issues with the single leader algorithms. Such solutions adopt the Generalized form of Consensus [21], which exploits the underlying commutativity of commands entering the system, such that the commutative commands can be ordered differently across different nodes and only noncommutative commands need consistent ordering across all the processes. Implementing this in the XFT model is nontrivial due to the addition of Byzantine nodes wherein commands originating from these nodes cannot be committed. Our goal is to provide a Generalized Consensus algorithm in the XFT model which achieves high performance in the geo-replicated setting using XFT, an adversary model which befits the geo-replicated setting.

\section{System Model and Problem Formulation}

This section specifies the system assumptions used for designing Elpis, the contribution of this paper. There exists a set $\Pi=\left\{p_{1}, p_{2}, \ldots, p_{N}\right\}$ of processes that communicate by message passing and do not have access to shared memory. Additionally, there exist clients which can communicate with any process in the system.

\subsection{Cross Fault-Tolerance (XFT) Model}

The processes may be faulty; they may fail by crashing $\left(t_{c}\right)$ or be Byzantine $\left(t_{n c}\right)$. However, faulty processes do not break cryptographic hashes, digital signatures, and MACs. A process that is not faulty is correct. The network is complete and each pair of processes is connected using a reliable point-topoint bidirectional link. The network can be asynchronous; that is, the processes might not be able to receive messages in a timely manner. In this case, we say that the network is partitioned and the system model abstracts these partitions as network faults $\left(t_{p}\right)$. Following the XFT model [24], the total number of faults are bounded by,

$$
t_{n c}+t_{c}+t_{p} \leq\left\lfloor\frac{N-1}{2}\right\rfloor
$$

where $t_{n c}$ are the number of non crash-faulty or byzantine processes, $t_{c}$ is the number of crash-faulty processes and $t_{p}$ is the number of partitioned processes. In any other case, the system is considered to be in anarchy. For discussion in this paper, the system is assumed to be never in anarchy - there always exists at least a majority of correct and synchronous processes.

The Generalized Consensus [21] specification is used where the processes try to reach consensus on a sequence of commands, the $C$-Struct. The Consensus algorithm orders noncommutative commands before deciding and decides commutative commands directly. Every process can propose commands using the $\mathrm{C}$-Propose interface and the processes decide command structures $C$-struct using the C-Decide $(C$ struct cs) interface. Finally, the identifiers for the objects accessed by the commands are known apriori and is represented with the $L S$ attribute in every command. That is, for a command $c$, the identifiers for its set of objects is $c . L S$.

\subsection{Problem Statement}

Given the system model, the problem is formulated as follows: How to implement State Machine Replication (SMR) using Generalized Consensus in the Cross Fault-Tolerance (XFT) model? The SMR clients invoke commands by sending a request to a process which then uses the C-Propose interface to propose. When the process decides, it applies the $C$-Struct to the State Machine and generates a reply which is returned to the client. Given that the majority of processes are correct and communicate synchronously (Equation 1), the following properties should be guaranteed.

Non-triviality Only proposed commands are decided and added to the $C$-structs.

Stability If a process decided a $C$-struct cs at any time, then it can only decide $c s \cdot \sigma$, where $\sigma$ is a sequence of commands, at all later times.

Consistency Two $C$-structs decided by two different processes are prefixes of the same $C$-struct.

Liveness If a command $c$ is proposed it will eventually be decided and added to the $C$-struct.

In Section 4, we illustrate how Elpis achieves State Machine Replication, and in Section 5, we prove that Elpis satisfies all of the properties listed above.

\section{Protocol Description}

\subsection{Overview}

Interestingly, Elpis derives the inspiration for implementing Generalized Consensus from $\mathrm{M}^{2}$ Paxos [28] which does not tolerate Byzantine faults. The core idea of $\mathrm{M}^{2} \mathrm{Paxos}$ is to 
avoid contention among multiple processes that propose noncommutative commands $C$ by dynamically choosing a unique owner for the objects on which the commands operate. This owner now orders all commands which access the objects for this epoch. Once an owner is chosen, other processes forward any command in $C$ to the owner. If a process does not have the ownership of the objects accessed by a command, it first tries to acquire the ownership by running the ownership acquisition phase (Section 4.3.5). If the process acquires the ownership, it tries to decide the command.

For Byzantine Fault Tolerant algorithms a quorum of size $2 t+1$ out of $3 t+1$ processes is required where $t$ is the number of faulty processes. The two Byzantine quorums intersect at $t+1$ processes, one of which is guaranteed to be correct. Elpis, on the other hand, uses $2 t+1$ processes. A set of $2 t+1$ processes would include $t$ faulty processes which is determinant to the liveness of the protocol. Hence, a quorum of size $2 t+1$ seems implausible. Elpis takes the approach wherein if a faulty process is detected, clients switch to another proposer after receiving $t+1$ Aborts in the commit phase (Section 4.3.3). Since a majority of processes are correct and communicate synchronously (Section 3.1), when an honest proposer is found this $t+1$ synchronous set of correct processes form a quorum of size $2 t+1$ with the $t+1$ processes in the iteration which last aborted. In the worst case the client retries request with a maximum of $t$ faulty proposers and on the $t+1$ try the request is decided.

The ownership acquisition can affect the progress of the protocol if multiple processes try to acquire the ownership by issuing an increasing sequence of epoch values similar to Paxos [19]. To attenuate this scenario all processes are allotted tag values picked from a set of totally ordered elements and prioritized as such when proposing. The acceptor which replies with a Nack message includes the tag of the process it last sent an Ack for the highest epoch for the objects in the message received. Upon receiving $t+1$ Nack messages the proposer starts a coordinated collision recovery phase by using a tag picked in a predetermined fashion (Section 4.3.2). At the end of the collision recovery phase (CR), the failing processes reevaluate the ownership configuration in the system depending on the result of CR and either take command of the objects or else forward the command to the process picked.

Initially, a home process $p_{i}$ that is closest (incurs lowest latency) to the client receives the request. The client sets a timer and waits for responses. Each correct process responds with either a signed Reply message or a signed Abort message. If the client receives $t+1$ messages with matching replies then the client is sure that the request is replicated. Alternatively, if the client receives $t+1$ Abort messages or the timer expires, then $p_{i}$ is not part of the correct and synchronous group and it retries with another process.

In summary, Elpis solves two challenges of implementing Generalized Consensus in the XFT model: (1) How to tolerate Byzantine faults with $2 t+1$ processes with no predetermined leaders (2) How to reliably acquire the ownership as measured in terms of the number of retries required in the presence of multiple processes vying to take the ownership of objects by using two major components:

1. A common-case protocol which allows processes to acquire ownership of the objects, decide the commands, and return responses to the clients.

2. A collision recovery protocol which helps resolve the ownership if multiple processes try to acquire the ownership concurrently.

\subsection{State maintained by a process $p_{i}$}

Each process $p_{i}$ maintains the following data structures.

- Decided and LastDecided The former is a multidimensional array that maps a pair of $\langle l, i n\rangle$ to a request where $l$ is the object and in is the consensus instance. Decided $[l][i n]=r$. If $r$ has been decided in the consensus instance in (i.e., in position $i n$ ) of the object $l$. The latter is a uni-dimensional array which maps the consensus instance in that $p_{i}$ last observed for an object $l$. The initial value for Decided is NULL while the initial value for LastDecided is 0 .

- Epoch It is an array that maps an object to an epoch number (non-negative integer). Epoch $[l]=e$ means that $e$ is the current epoch number that has been observed by $p_{i}$ for the object $l$. The initial value is 0 .

- Owners. It is an array that maps an object to a process. Owners $[1]=p_{j}$ means that $p_{j}$ is the current owner of the object 1 . The initial values are NULL.

- Rnd, CommitLog, StatusLog These are three multidimensional arrays. The first one maps a pair of $\langle l, i n\rangle$ to an epoch number. $R n d[l][i n]=e$ if $e$ is the highest epoch number in which $p_{i}$ has participated in the consensus instance in of object $l$. Therefore, CommitLog $[l][i n]=\langle r, e\rangle$ implies that the process received a quorum of Commits for request $r$ and epoch $e$. The StatusLog maintains the valid $\langle r, e\rangle$ that the process is willing to commit on. Hence, StatusLog $[l][i n]=\langle r, e\rangle$ implies that $p_{i}$ would accept a replicate message for $r$ in epoch $e$ and reject others.

- statusList, commitList, decideList, trustList. These are four multidimensional arrays which are used to store Сомміт, Status, Decide and Trust messages respectively. The initial value is NULL.

- Tags An array which maps a process $p_{i}$ to it is tag. The tag of a process $p_{i}$ is equal to $\operatorname{Tag}\left[p_{i}\right] \in S$ where $S$ is a totally ordered set. The tag is used during collision recovery (Section 4.3.6). This mapping has to be predefined by the application layer during setup and is static during the protocol execution.

- Estimated A multidimensional array which maps the $\langle l, i n\rangle$ to the address of the process which this process estimates to be the owner of the object 1 for an epoch e. Hence, Estimated $[l][i n]=\left\langle e, t_{p_{e}}, p_{e}\right\rangle$ implies that for epoch $e$ this 


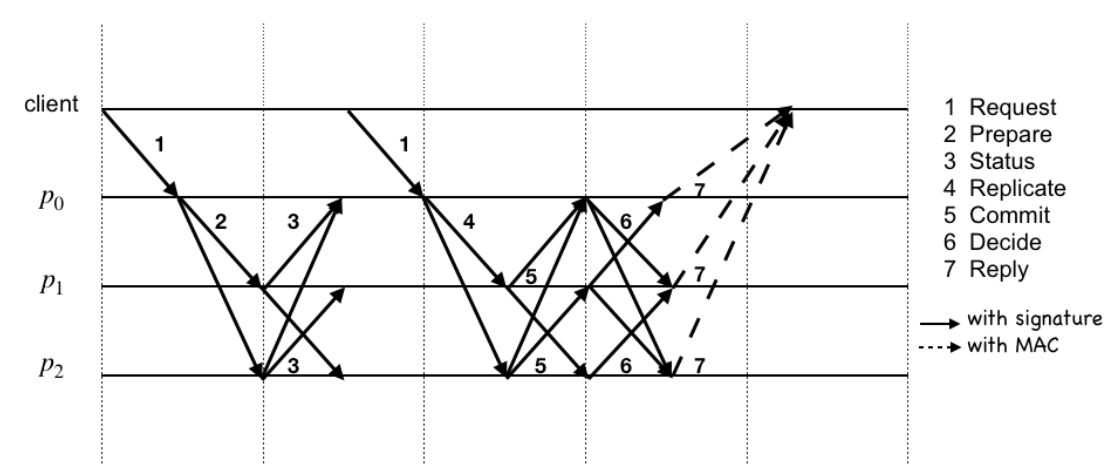

Figure 1. Elpis: $p_{0}$ sends a Prepare first to acquire the ownership or Replicate directly if it has the ownership of all the objects in the request. Client expects $t+1$ matching Reply messages.

process estimates $p_{e}$ to be the owner where $t_{p_{e}}$ is the tag of the process.

- Leader This is a multidimensional array which maps the $\langle l, i n\rangle$ pairs to the $\left\langle e, p_{t}\right\rangle$ pairs for which the collision recovery decides ownership. The value of this array is updated only during the collision recovery. The initial value is NULL.

\subsection{Detailed Protocol}

It is assumed that all processes, including the clients, possess public keys $P_{k}$ of all the processes. Each message $m$ includes the digest of the message $D(m)$ and a signed message sent by some process $p$ along with it is digest is represented as $\langle m\rangle_{\sigma_{p}}$. Unless otherwise stated, each process validates the messages received by first verifying the signatures using the corresponding public key in $P_{k}$ and then by verifying the message by using a checksum mechanism by comparing it against the message digest. Any message parameter which includes object $l$ as the key can be verified to be for the correct $l$ by matching the objects in req.LS. In other words, an object $l^{\prime}$ cannot exist in the message which does not exist in req.LS, otherwise the message is deemed to be invalid.

A client $c$ sends a signed request $r e q=\langle\operatorname{ReQUEST}, o, t, l s, c\rangle_{\sigma_{c}}$ to a process $p_{i}$ where $o$ represents the command to be executed, $t$ is the client's timestamp, and $l s$ contains the objects accessed by the operation $o$ and sets a timer. The timer is useful if the client sends a request to a process which has crashed or is partitioned from other processes.

\subsubsection{Coordination Phase}

When a request req is proposed by process $p_{i}$ using the C-Propose interface, Elpis coordinates the decision for req. In the Coordination phase (Algorithm 1), $p_{i}$ reads the ownership of objects in the system. Depending on the current ownership configuration, the process either invokes the replication phase (Section 4.3.2), forwards the request to the owner or tries to acquire the ownership for all the objects accessed by the req by executing ownership acquisition (Section 4.3.5).

The process $p_{i}$ finds the consensus instance it last decided for each object in $L S$ and which is not decided for req. For every such object, $p_{i}$ sets $i n$ equal to LastDecided $[l]+1$ and

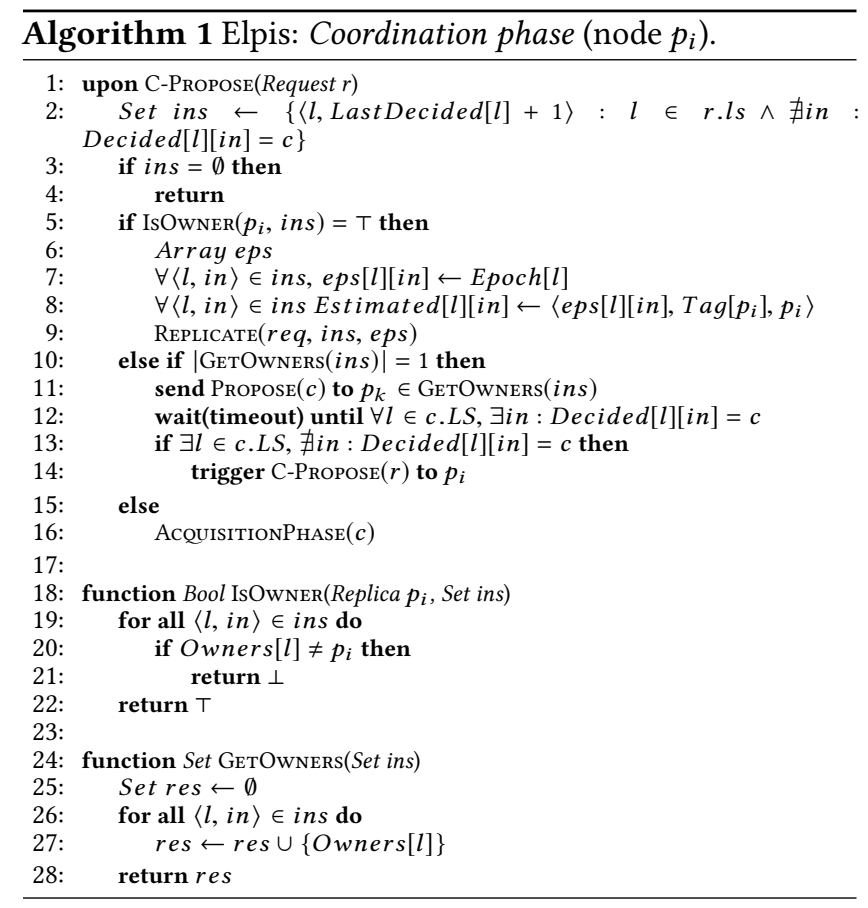

adds it to the ins set (line 2). If the process has the ownership of all objects in req.LS then the process tries to achieve a fast decision by executing the replication phase without changing the epoch. If the replication phase succeeds, $p_{i}$ is able to execute the req in two communication delays and returns the response to the client.

Alternatively, If $p_{i}$ detects that $p_{k}$ has the ownership of all objects in ins, it forwards the req to the $p_{k}$. To avoid blocking in case $p_{k}$ crashes or is partitioned, $p_{i}$ also sets a timer. Upon expiration of the timer, if the $p_{i}$ detects that the req has not been decided, it takes charge of the req and tries to $C$ - Propose the req (lines 10-14).

Finally, if $p_{i}$ detects no owners for all objects in ins, it tries to acquire the ownership by executing the acquisition phase (4.3.5) (line 14). A different process, $p_{k}$ can have the ownership of some subset of objects in req.LS, however this process proceeds to steal ownership as complete ownership 
is necessary for setting the correct instance number ins for proper linearization of commands during execution.

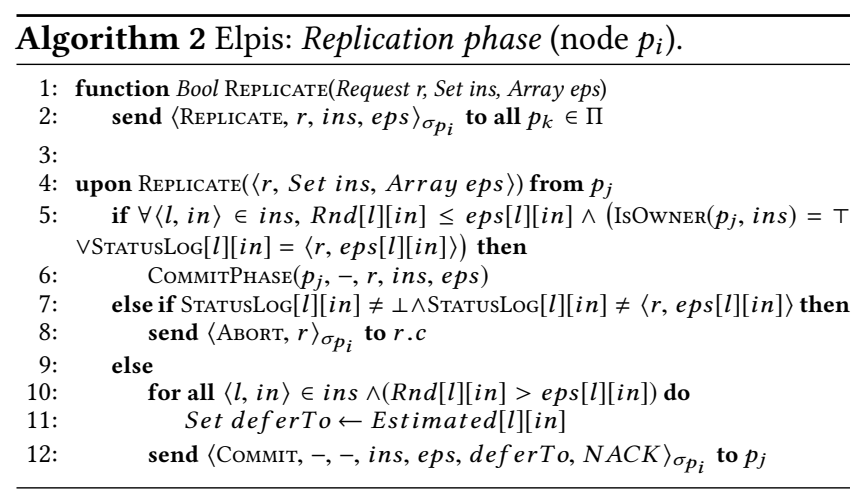

\subsubsection{Replication Phase}

In the Replication phase (Algorithm 2), $p_{i}$ requests the replication of request req for instance ins and epochs eps. It sends a signed REPLICATE message to all processes in $\Pi$. Upon receiving a REPLICATE message the process $p_{j}$ checks if the received message is for an epoch greater than or equal to the last observed $R n d[l][i n]$ for all the objects in the request and checks if $p_{i}$ is, in fact, the owner of all the objects in the message (line 5 ). If both of these conditions are satisfied, $p_{j}$ starts the commit phase (Section 4.3.3) for the request with the received ins and eps values (line 6).

The Replication phase is invoked during either the Acquisition phase (Section 4.3.5) where a process is trying to acquire the ownership or invoked directly by a process which already has the ownership of all objects in the request req.LS. Otherwise, a StatusLog is constructed by collecting Status messages in the Acquisition phase. This request, epoch pair in the StatusLog $[l][\mathrm{in}]$ is considered to be valid. This is discussed further in (Section 4.3.5). Hence, the $\langle r, e p s[l][i n]\rangle$ in the REPLICATE message should match the values in the StatusLog for a process $p_{i}$ which is trying to acquire the ownership. If this is not the case then $p_{i}$ has equivocated and hence, this phase concludes by sending an AвоRт message to the client.

Otherwise, if the message does not fall under either of the cases mentioned above then $p_{j}$ has already acknowledged a message for eps[l][in] from the owner of the objects in the message and sends a Nack message along with the information about the last process it sent an Ack for the highest epoch for one or more $\langle l, i n\rangle$ pairs (lines 8-10). This information returned with the Nack message is relevant for the collision recovery and it is discussed in detail in Section 4.3.6.

\subsubsection{Commit Phase}

In the Commit phase (Algorithm 3), correct processes coordinate to pick a valid request for instances in ins and for the epochs in eps in the presence of Byzantine processes.
The request req received in the REPLICATE message is broadcasted using Cомміт messages and each received CоммIт is collected in the commitList (lines 11-14).

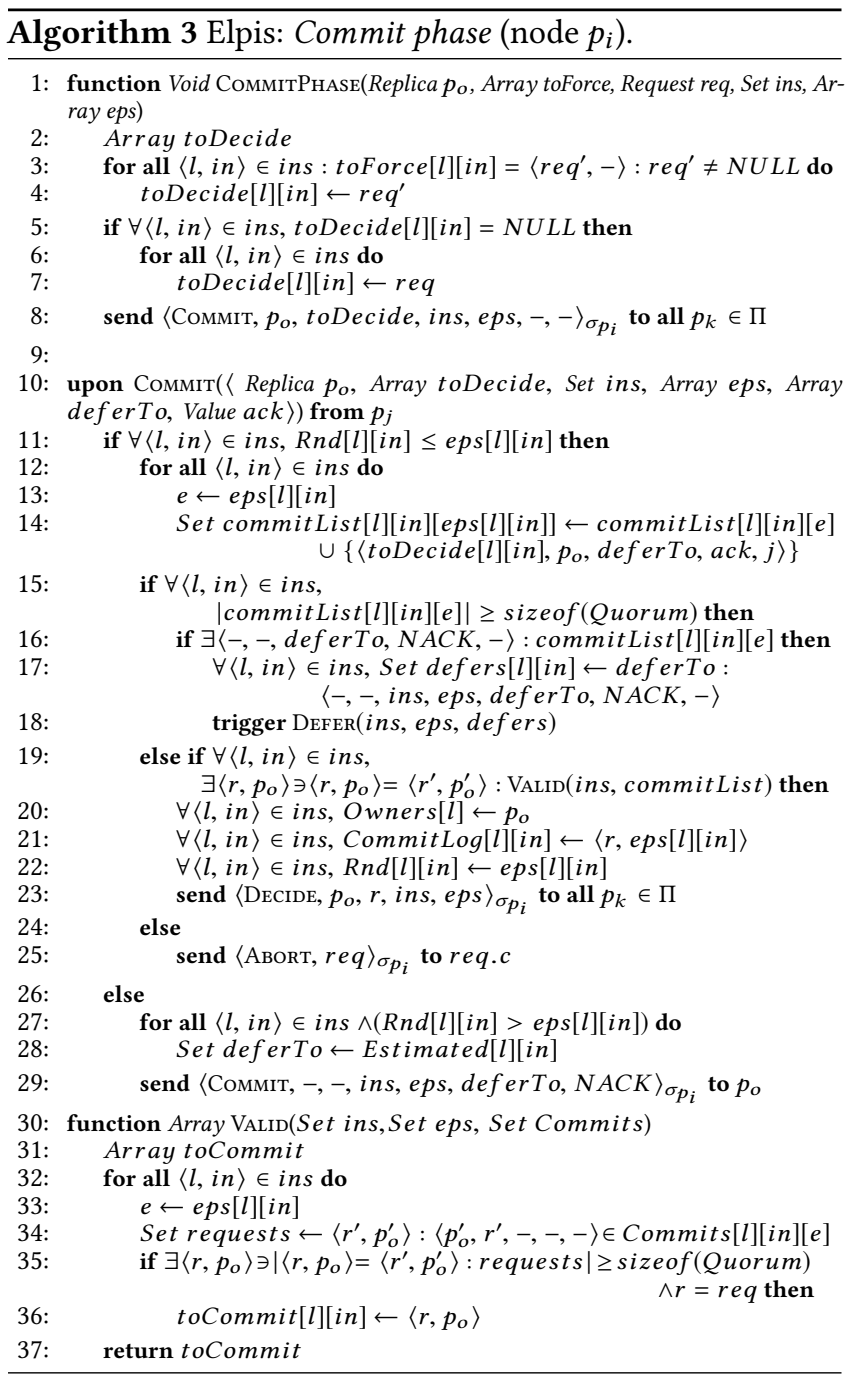

There are two cases for Byzantine processes: (1) Any $t$ acceptors could send arbitrary request values rather than forwarding req, (2) The proposer which has the ownership can equivocate by sending req to some processes and some request $r e q^{\prime}$ to the rest of the processes. To tackle both of these scenarios a request $r$ is valid if $p_{i}$ receives $t+1$ matching Cомміт messages (Ack) for $r$ and $r$ matches the request for which this phase was invoked. If there exists a valid req for all $\langle l, i n\rangle$ pairs, then this phase successfully concludes by setting the owners array to the process which sent the REPLICATE message which invoked this Commit phase, adds the values to the CommitLog and sends a DECIDE message to all the processes (line 19 - 22). Otherwise, if either there exists no common request req in atleast $t+1$ messages or $r e q$ does not match the request for which this phase was invoked, 
then this process Aborts by sending an Abort message to the Client (line 24).

If $p_{i}$ does not acquire $t+1$ Commit messages $(A c k)$ and there exist Nack Commit messages, then some other process has stolen the ownership. In this case, $p_{i}$ triggers the Collision Recovery phase (Section 4.3.6).

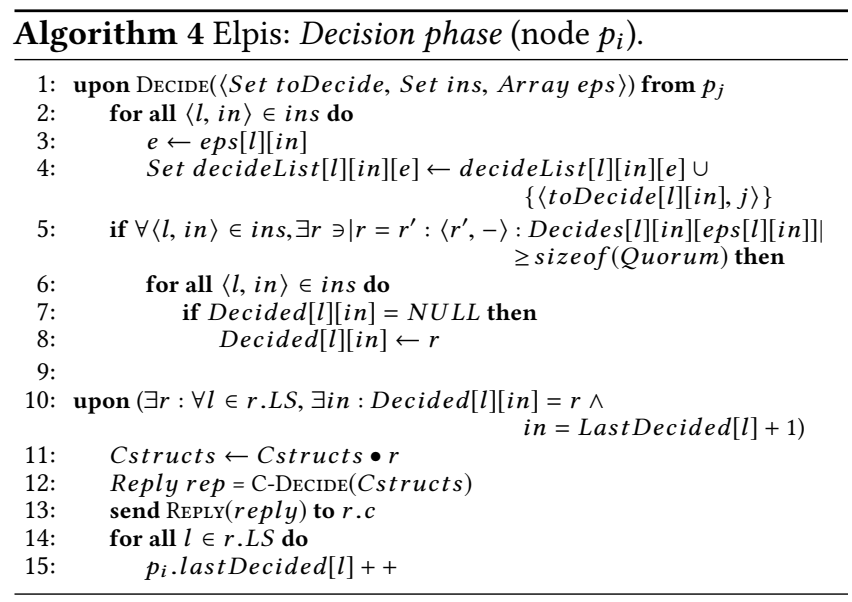

\subsubsection{Decision Phase}

In the Decision phase (Algorithm 4) a process $p_{i}$ tries to learn a request. Upon receiving a DEcide message the process stores the message in the decides array indexed by the $\langle l, i n\rangle$ pair and the epoch $e$. If $p_{i}$ receives $t+1$ matching messages then the process $p_{i}$ assumes this request to be decided for the object $l$ and instance in (lines 2-6). When a request is decided for all the objects accessed by the request, $p_{i}$ appends it to its Cstruct, executes the request and returns the response to the client as a REPLY message and increments the LastDecided for all objects (lines 7-13).

\subsubsection{Acquisition Phase}

In the Acquisition phase (Algorithm 5) the process $p_{i}$ tries to acquire the ownership of the objects in req.ls and also assure that a faulty process is not able to acquire the ownership.

Similar to the Coordination phase, for each object in $l s$ of the request req the process $p_{i}$ finds the consensus instance LastDecided $[l]$ it last decided for the object and which is not decided for $c$ and finds the next position by setting in equal to LastDecided $[l]+1$ and adds it to the ins set. Additionally, for each pair $(l, i n) \in$ ins, it increments the current epoch number for the object $l$. The process $p_{i}$ now sends a Prepare message to all processes in $\Pi$ (lines 1-6).

Before sending a Prepare message, the process also sets Estimated $[l][$ in $]$ to its tag and epoch thus estimating itself to acquire the ownership. This is relevant if this process receives a Prepare message for the same or a lower epoch for the objects it is trying to acquire the ownership. In that case, this process will send a Nack message using the Estimated $[l][$ in $]$ values.

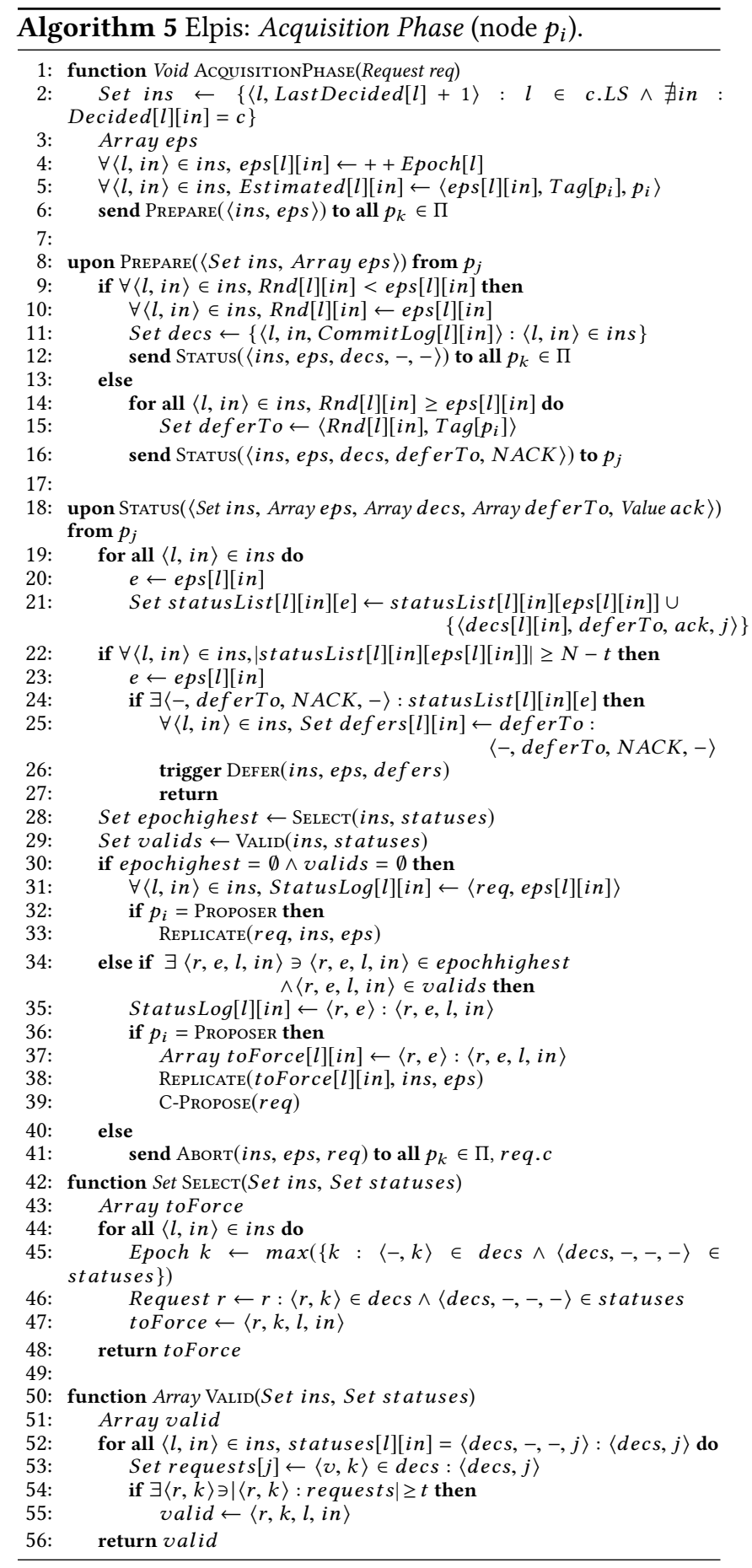

Upon receiving a PREPARE message with a higher epoch for all objects than the last observed, each process sends its CommitLog in the STATUs message to all the processes (lines 8-12). If the received message has a lower epoch, it sends a Nack message with the information about process it last sent an Ack for (lines 14-16). The STATUs message includes the CommitLog for the (object, instance) pairs. Upon receiving STATUs messages from enough processes (at least $N-t$ ), the 
process decides if there is a request to be committed from an aborted Commit Phase from an earlier epoch. For this, for all $\langle$ request, epoch〉 entries present in the CommitLog received from a process $p_{j}$, the process first calculates the highest epoch values present in the entries for which a request is present and adds such $\langle$ request, epoch $\rangle$ pairs to the epochhighest set (lines 20-27).

However, the request in this log could be from a Byzantine process. To eliminate such requests, each process also calculates a valid $\langle$ request, epoch $\rangle$ pair by reading the CommitLogs it received as part of the Status message. If a pair is present in more than the number of faulty processes then this $\langle$ request, epoch $\rangle$ pair is validated. If a $\langle$ request, epoch $\rangle$ is present in the epochhighest set and is also present in the validated set then process starts a commit phase with a toForce array which contains this $\langle$ request, epoch $\rangle$ pair.

However, if it is not present in either of those sets then the process starts the Commit Phase with an empty array. If however, a pair exists in the quorumhighest set and is not present in the validated set or vice versa the leader has equivocated and the phase Aborts by sending an AвоRт message to all processes including the client. Upon receiving $t+1$ such AвоRт messages the client retries the request with a different process.

\subsubsection{Collision Recovery}

Collision recovery (Algorithm 6) is used to reduce the number of processes contending to acquire the ownership of some object(s). When a process $p_{i}$ receives Nack messages (line 24 in Algorithm 5 and line 16 in Algorithm 3), the process $p_{k}:\left\langle-, \operatorname{tag}, p_{k}\right\rangle \in$ deferTo is a process which is executing Elpis for an epoch equal to or higher than the Epoch $[l]$ at $p_{i}$ for some object $l$. Hence, some subset of acceptors return a Nack message as they have already sent an Ack to process $p_{k}$. There could be multiple processes like $p_{k}$ at any given time. For instance, if all processes propose simultaneously for the same epoch. This phase provides a coordinated mechanism to find a process which is executing ownership acquisition for the highest epoch with any ties broken by using the tags of the processes. Succinctly stated, $p_{i}$ uses collision recovery to conform to the current ownership reconfiguration taking place in the system as opposed to contending by proposing higher epoch values.

The process $p_{i}$ may receive multiple Nack messages. In this case, a set of rules (similar to Fast Paxos [22]) are used where $p_{i}$ tries to PICK a process to defer to. A deferTo value is picked if it exists in a majority of Nack messages or has the maximum count with any ties broken by using the tags. After the completion of the Collision recovery (CR), the process sets its owners to the one learned from CR and retries the request with the coordination phase (lines 1-5).

Before starting the phase $p_{i}$ checks if an instance of Collision Recovery has already been completed by the system (invoked by some other process). In this case, no additional

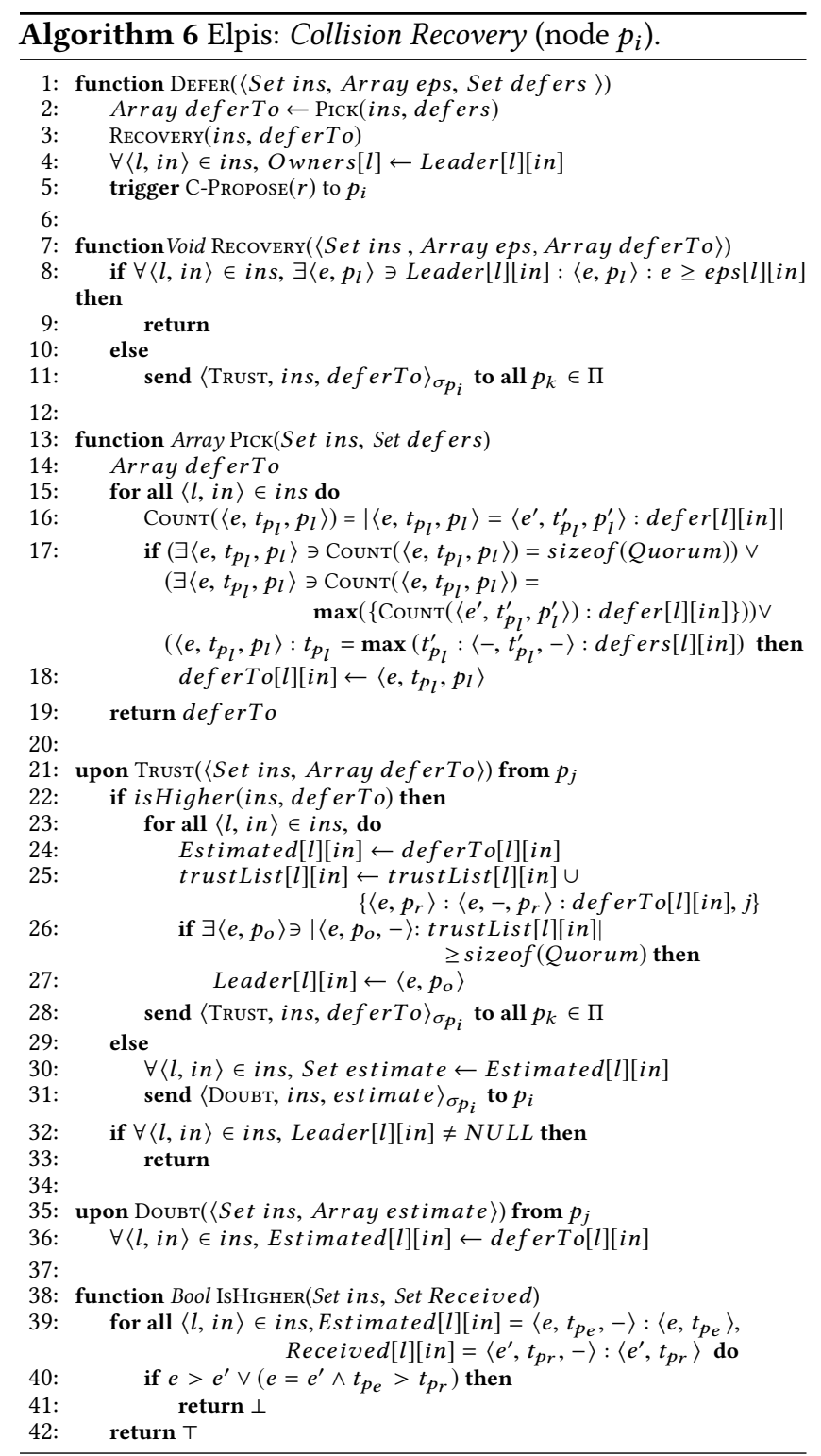

run is required and $p_{i}$ concludes the recovery (line 8-9). However, if no such instance has been completed then $p_{i}$ start this recovery by sending a $\langle\text { TRUST, ins, eps, leader }\rangle_{\sigma_{p_{i}}}$ message to all the nodes (line 5).

Upon receiving a TRUST message the process compares the current estimated leader value to the received value. The values are ordered by using their epochs first and then by their tags. That is, a value is Higher if it has a higher epoch. If the epochs are equal then the node tags are used to break the symmetry (lines 38-42). Therefore, if the received value is Higher, then the process sets this as the new estimated value, stores the value in its trustList and forwards the TRUST message to all the processes with the received value (lines 22-26). If the value is lower however, the process sends a DoubT message with the higher value (line 30-31). 
Upon receiving a DouBT message the process sets its Estimated to the value received in the defer message (line 22). When the cardinality of statusList $[l][$ in $]$ equals the QuORUM for some $\langle l, i n\rangle$ pair then the process $p_{l}:$ trustList $[l][i n]$ is trusted to be the owner of this $\langle l, i n\rangle$ pair and when there is a trusted owner for all $\langle l, i n\rangle \in$ ins then the recovery concludes.

\section{Correctness}

We formally specified Elpis in TLA + [20], and model-checked with the TLC model checker for correctness as a decision on the correct value despite the presence of Byzantine acceptors and abort when the proposer equivocates. The TLA+ specification is provided in two anonymous technical reports ${ }^{1,2}$ for the algorithm component and the collision recovery component. In this section, we provide an intuition of how Elpis satisfies the protocol's guarantees.

Stability: Only the owner of an object $l$ in epoch $e$ successfully commits the requests, and thus increments in. A Byzantine process does not acquire ownership as the proposer equivocation is detected and the execution is aborted. Since, the correct processes initially start with the same value of LastDecided $[l]$ and only increment it when a command is decided for $\langle l, i n\rangle$, the valid requests proposed by a correct owner of $l$ in $e$ would follow a complete order for in throughout the execution of the protocol and would not diverge for any correct process.

In the rest of the section we refer to StatusLog[l][in] and CommitLog[l][in] as StatusLog and CommitLog for brevity which denote the value of the logs for some $\langle l, i n\rangle$. The proofs can be generalized for any instance in of the object for which the process acquires ownership of the object $l$.

Non-triviality: A process only appends a command $c$ to the $C$-Struct if it receives Commit messages from a majority of processes for $c$ and no other command can exist. Correct processes only send Commit messages for the value $c$ if they receive $c$ in the Replicate message.

Consistency: Lets assume some process $p_{i}$ decides a command $c$ for some $\langle l, i n\rangle$ and epoch $e$. This must imply that this process received Decide messages from $\geq \frac{N}{2}$ processes with the command $c$ and $\langle l, i n\rangle$ and epoch $e$. Hence, there must be a set $X$ of size $\frac{N}{2}>t$ which received $\geq \frac{N}{2}$ Commit messages for the command $c$ for $\langle l, i n\rangle$ and epoch $e$. All processes in $X$ set CommitLog $=\langle c, e\rangle$. The state of at least one correct process is contained in the quorum and because all processes in $X$ include $\langle c, e\rangle$, the StatusLog $=\langle c, e\rangle$ in the next epoch.

We argue that if a correct process in $X$ commits request $c^{\prime}$ in the epoch $e^{\prime}$, and StatusLog $=\langle c, e\rangle$ then for $e^{\prime}:\left\langle c, e^{\prime}\right\rangle$ $=$ StatusLog $\wedge e^{\prime} \geq e, c^{\prime}=c$. We prove this by induction on the epoch $e^{\prime}$. For the base case, lets suppose StatusLog $=$

\footnotetext{
${ }^{1}$ Elpis TLA+ Specification: http://bit.ly/elpistla

${ }^{2}$ Elpis Collision Recovery TLA+: http://bit.ly/elpiscr
}

$\left\langle c, e^{\prime}\right\rangle$ at some correct process $p_{i}$. If $p_{i}$ commits $c$ in $e^{\prime}$ then it must receive a Replicate request for $c$. Otherwise if it receives a request for $c^{\prime} \neq c$ it would detect that the process contending for ownership has equivocated and Abort. Hence, $c^{\prime}=c$. For $e^{\prime}, p_{i}$ commits on $c^{\prime}$, sets CommitLog $=\left\langle c^{\prime}, e^{\prime}\right\rangle$ sets the process which sends $c^{\prime}$ as the owner (which is in fact correct).

Lets suppose that for any epochs in between $e^{\prime}$ and $e$, StatusLog $=\left\langle c^{\prime},-\right\rangle$. We have to prove that if StatusLog $=$ $\langle c, e+1\rangle$ then $c=c^{\prime}$. The StatusLog $=\langle c, e+1\rangle$ consists of valid CommitLogs for $c$ in $e$. Since, the StatusLog $=\left\langle c^{\prime}, e\right\rangle$ any correct process that commits $c$ and sets its Commit Log to $\langle c, e\rangle$ can only do that if $c$ and $c^{\prime}$ are equal. Hence, $c=c^{\prime}$. By induction we can say that this is true for all $e^{\prime} \geq e$.

We use this argument to prove agreement: If two correct processes commit $c$ and $c^{\prime}$ then $c=c^{\prime}$. If a correct process initially commits $c$ in $e$, then StatusLog $=\langle c, e\rangle$. If another correct process commit $c^{\prime}$ in $e^{\prime}$, then we know that for any $e>e^{\prime}, c=c^{\prime}$. Hence, the correct processes must agree.

Liveness: Under the assumptions of the XFT model, there always exists at least a majority of processes that are correct and synchronous. We see that in the case of a malicious leader, every correct process detects equivocation and sends Abort messages to the client. After receiving $t+1$ messages the client switches to a new process. If the process is Byzantine it would again receive the Abort messages or timeout. This can only happen a maximum of $t$ times.

We show liveness by proving that a correct process in $e^{\prime}(>e)$ is able to collect $N-t$ Status messages and calculate a StatusLog to find the $\langle c, e\rangle$ values. If a CommitLog contains a value $\langle c, e\rangle$, this means it must have received Commit messages for this value from $\frac{N}{2}$ processes (line 21 Algorithm 3). Since, every correct node broadcasts Commit messages, it's easy to see that all $\frac{N}{2}>t$ nodes in the system contain the same value in their CommitLog as well. Using this and the consistency property above we can see that in round $e^{\prime}$, if a correct process $p_{i}$ receives the client request, then every correct process is able to collect the same $N-t$ CommitLogs and set StatusLog to $\langle c, e\rangle$. The process $p_{i}$ now sends a value matching the StatusLog. At least $t+1$ processes share Commit messages and Decide the value.

\section{Evaluation}

We evaluate Elpis by comparing it against four other consensus algorithms: XPaxos, PBFT, Zyzzyva and $\mathrm{M}^{2} \mathrm{Paxos}$. We take the latency measurements in a geo-replicated setup by setting up seven nodes using Amazon EC2 (Table 1) and throughput by placing the nodes in a single placement group us-east-1 so as to avoid skewing the data due to a greater variance in latencies in case of the geo-replicated setup. Additionally, all the clients are placed at respective nodes to simulate real-world implementations where requests are served by the closest data center. 


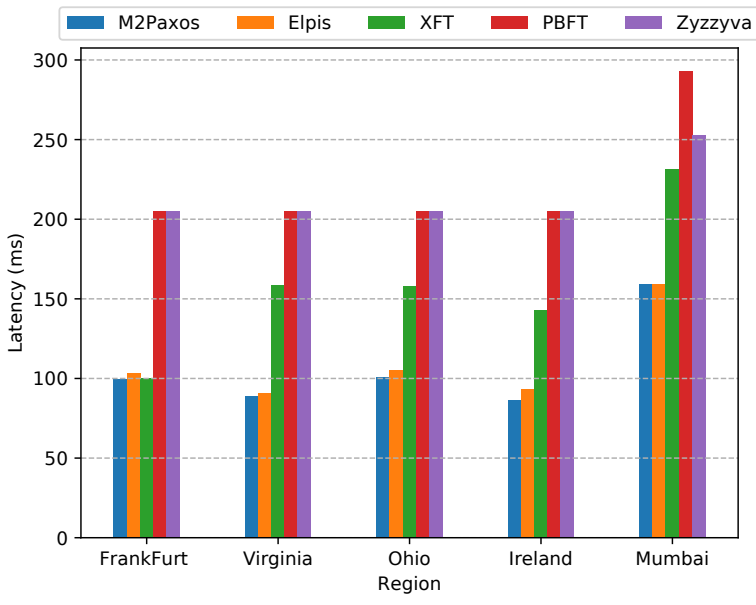

(a) 5 nodes

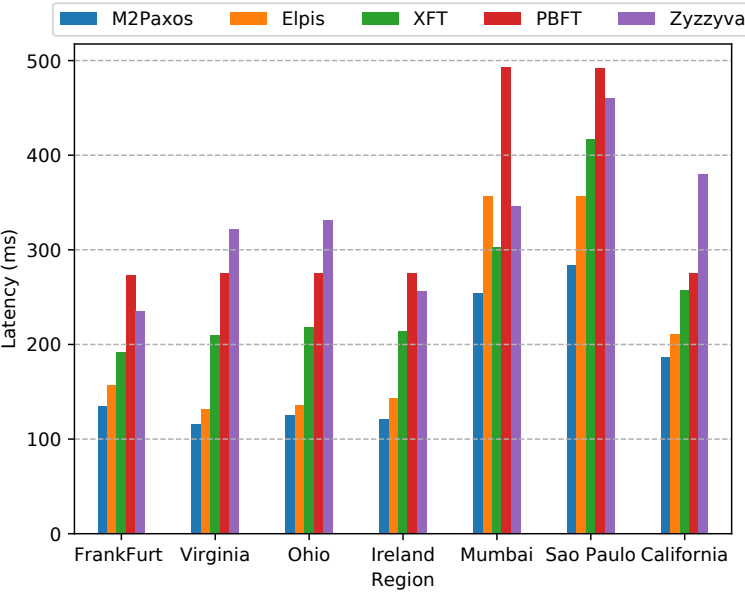

(b) 7 nodes

Figure 2. Latency comparison across regions.

We implemented Elpis, XFT and $\mathrm{M}^{2}$ Paxos using the reliable messages toolkit Jgroups [9], in Java 8. We used the ClusterPartition MBean configuration and leveraged ASYM_ENCRYPT protocol configured with RSA 512 for asymmetric and AES/ECB/PKCS5Padding with 128 bit key size for symmetric encryption for Elpis. We implemented Zyzzyva using the BFT-SMaRt library (also in Java 8) and used the default highly optimized PBFT implementation. Unless otherwise stated, each node is a c3.4xlarge instance (Intel Xeon 2.8GHz, 16 cores, 30GB RAM) running Ubuntu 16.04 LTS Xenial (HVM).

\begin{tabular}{|l|l|c|c|c|c|c|c|c|}
\hline AWS Region & Name & Virginia & Ohio & Frankfurt & Ireland & Mumbai & California & Sao Paulo \\
\hline us-east-1 & Virginia & - & 11 & 88 & 74 & 182 & 59 & 140 \\
\hline us-east-2 & Ohio & 10 & - & 97 & 84 & 191 & 49 & 149 \\
\hline eu-central-1 & Frankfurt & 88 & 97 & - & 21 & 109 & 145 & 226 \\
\hline eu-west-1 & Ireland & 74 & 84 & 39 & - & 122 & 129 & 183 \\
\hline ap-south-1 & Mumbai & 182 & 191 & 109 & 120 & - & 241 & 320 \\
\hline us-west-1 & California & 59 & 49 & 145 & 129 & 241 & - & 197 \\
\hline sa-east-1 & Sao Paulo & 140 & 149 & 226 & 183 & 320 & 197 & - \\
\hline
\end{tabular}

Table 1. Average inter-region RTT latencies in ms.

\subsection{Experimental Setup}

For PBFT, Zyzzyva, and XPaxos the primary is placed at Frankfurt. Additionally, the initial synchronous group for XPaxos consists of \{Frankfurt, Ireland, Ohio $\}$ and \{Frankfurt, Ireland, Ohio, Virginia\} for the five node and seven node experiment respectively. For processes in the single placement group of Virginia the latency for communicating with other processes in the group was observed to be close to $2 \mathrm{~ms}$. To properly load the system, we injected commands into an open-loop using client threads placed at each node. Commands are accompanied by a 16-byte payload. However, to not overload the system we limit the number of in flight messages by introducing a sleep time where every client sleeps for a predetermined duration after proposing a request. This is tuned so as to get the best possible performance for the setup. We implemented a synthetic application that generates a workload which covers partitionable case with no inter-node conflicts (objects are locally accessed), to when command forwarding is required (a remote owner present for the objects), and to when multiple nodes have to acquire the ownership. Since, we are just testing the Consensus layer we do not execute any commands.

\subsection{Latency}

Figure 2 shows the comparison of latencies in a geo-replicated setup where the requests have $100 \%$ locality which implies that the requests in different regions access different objects. We notice that $M^{2}$ Paxos achieves the best response time for all regions due to a lower quorum size. Elpis expectedly achieves close but slightly higher latencies than $\mathrm{M}^{2}$ Paxos due to the additional overhead of message digests and message broadcasts. This overhead is inherent to all the other protocols including XPaxos. XPaxos achieves best response time for Frankfurt (the primary). For clients present in all the other regions the request forwarding to Frankfurt results in higher response times. In contrast, the primary/owner for every client in the case of Elpis is present in the same region as the client, which provides lower response time. PBFT and Zyzzyva latencies are much higher due to the same reasons as XPaxos compounded by the requirement of a greater quorum size. Hence, at each step, the primary has to wait for more messages and thus incurs longer response times. In summary, Elpis achieves response times close to $\mathrm{M}^{2}$ Paxos while promising better resilience.

\subsection{Throughput}

Figure 3 shows the throughput comparison in the single placement group us-east-1 as the system is pushed closer to saturation to achieve the maximum throughput possible. Elpis- $\mathrm{x}$ shows the performance under $\mathrm{x} \%$ conflict where $\mathrm{x} \%$ 


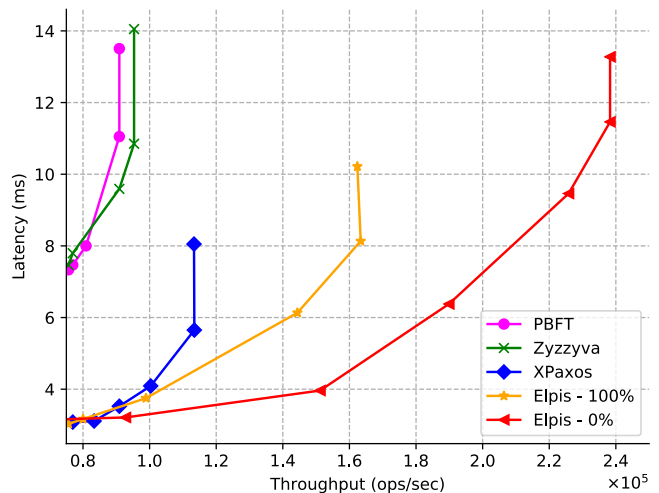

Figure 3. Latency vs. throughput in a cluster.

implies that the commands issued by a node access objects out of which $\mathrm{x} \%$ are shared with all the other nodes. Hence, Elpis-0\% implies no conflict and Elpis- $100 \%$ implies that all the clients across all nodes propose commands that access shared objects. PBFT and Zyzzyva peak at under $1 \times 10^{5} \mathrm{op}-$ erations/sec due to complicated message patterns resulting in higher bandwidth usage. XPaxos and Elpis perform significantly better as they replicate requests to lower number of followers as compared to Zyzzyva ( $t$ acceptors vs all $3 t$ nodes) and have less communication steps as compared to PBFT. Since Elpis relies on multiple owners the inherent load-balancing in the protocol results in higher throughput as compared to XPaxos where the primary becomes a bottleneck. As such even the $100 \%$ conflict case achieves higher throughput than XPaxos because for Elpis all the nodes are active as compared to XPaxos where only three (the active synchronous group consists of $t+1$ nodes for XPaxos) participants are active.

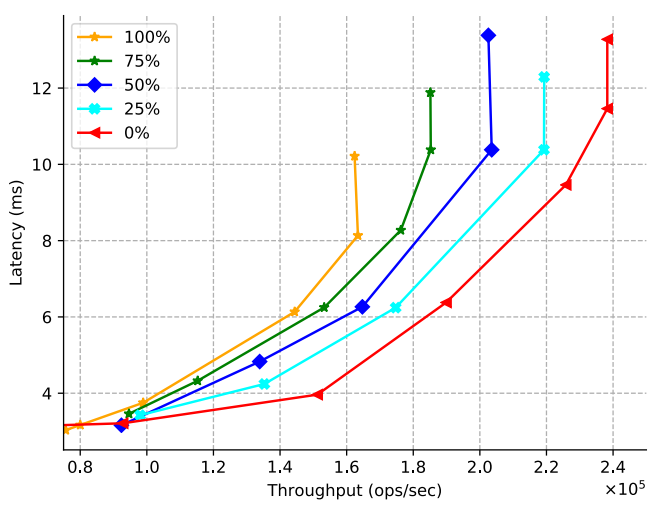

Figure 4. Performance under contention.

\subsubsection{Performance under contention}

Figure 4 shows the throughput vs latency comparison for Elpis as the percentage of contention is varied from $0 \%$ to $100 \%$. Throughput decreases as the percentage of contention increases. In the case of contention, collision recovery is invoked which increases the number of messages exchanged.
Hence, the network is stressed until a single owner emerges for each object for the conflicting commands which orders all these commands. Hence, a lower percentage of requests are concurrently executed across nodes. However, even in the presence of $100 \%$ contention Elpis outperforms all the competitors as shown in Figure 3.

\subsubsection{Performance under faults}

In this section, we show the behavior of Elpis in the presence of faults $(t)$. Figure 5 shows throughput as a function of time. A Byzantine process is simulated by adding a Byzantine layer in the Jgroups protocol stack under the Elpis implementation which when activated intercepts the RePLICATE messages and changes the req value in the message to an arbitrary value. At time 40 secs the message interception is activated at one of the nodes. This Byzantine node is detected by the other nodes and the clients start forward their requests to a different node after receiving $t+1$ AвоRт messages. The

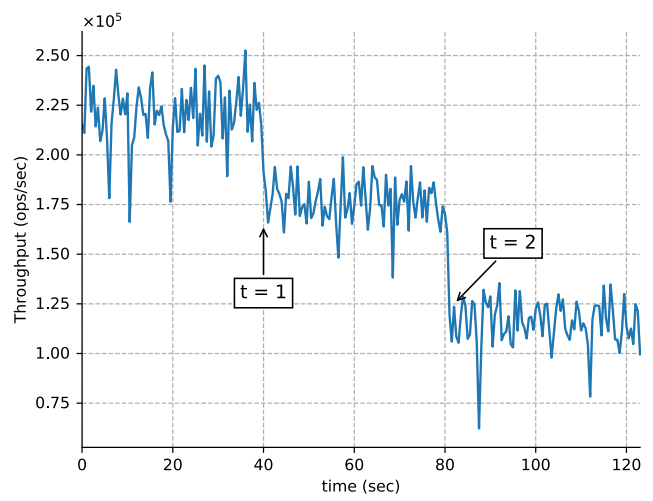

Figure 5. Performance under faults. Initially, $t=0$.

node to which the request is forwarded is pre-configured for this experiment. As a result, throughput is decreased as this node is no longer completing the requests however, the remaining nodes continue to serve the client requests. In the presence of a single fault PBFT, Zyzzyva and XPaxos would start a view-change as a result of which the throughput would be effectively reduced to zero as no requests can be processed until a new leader emerges. At time 80 secs, message interception at another node is triggered. At this point, Elpis continues to decide the client requests via the active nodes while tolerating the maximum number of faults outside anarchy.

\section{Related Work}

PBFT [15] was the first efficient solution to solve consensus in the BFT model. The protocol requires $3 t+1$ processes to tolerate $t$ faults and uses a quorum of size $2 t+1$ to return the result in five message delays. Zyzzyva [18] requires the same number of processes but achieves consensus in three message delays when no processes are slow or faulty. As such it requires bigger quorums of $3 t+1$ for the single-phase 


\begin{tabular}{|l|c|c|c|c|c|}
\hline Protocol & PBFT & Zyzzyva & $\mathbf{M}^{2}$ Paxos & XPaxos & Elpis \\
\hline Resilience & $t<n / 3$ & $t<n / 3$ & $t^{\star}<n / 2$ & $t<n / 2$ & $t<n / 2$ \\
\hline Best case communication steps & 5 & 3 & 2 & 2 & 3 \\
\hline Base case in the absence of & $t_{n c}$ & $t_{n c}, t_{p}$, contention & $t_{c}, t_{p}$, contention & $t_{n c}, t_{c} t_{p}$ & $t_{n c}, t_{c}, t_{p}$, contention $^{\dagger}$ \\
\hline Slow-path steps during contention & - & 2 & 2 & - & 2 \\
\hline Leader & Single & Single & Multi-Leader & Single & Multi-Leader \\
\hline Fault Model & BFT & BFT & CFT & XFT & XFT \\
\hline
\end{tabular}

Table 2. Comparison of existing protocols and Elpis. $\star$ only tolerates crash faults $\dagger$ may require multiple runs of this step.

execution in contrast to $2 t+1$ required for PBFT. Zyzzyva is not particularly suited for heterogeneous networks like the ones in geo-replicated systems as even a single constrained network link at any node can make it switch to a slower two-phase operation. Furthermore, these protocols rely on a single leader which is a bottleneck for throughput in geo-replicated systems during normal operation and incurs downtime during view-change.

Elpis treats $\mathrm{M}^{2} \mathrm{Paxos}$ [28] as an extended specification of Generalized Consensus and inherits a portion of data structures and interfaces. Elpis manages dependencies by mapping an object $o$ to a process $p_{o}$ similar to $\mathrm{M}^{2} \mathrm{Paxos}$. However, it innovates on how it manages contention. Agreeing on ownership of $o$ is a consensus problem in itself and $\mathrm{M}^{2}$ Paxos uses a mechanism similar to Phase 1 of Paxos [19]. As such, it does not guarantee liveness when multiple processes try to propose commands concurrently. This becomes even more evident when there exist cyclic dependencies in compound commands (that access multiple objects) such as $C_{1}:\{a, b\}, C_{2}:\{b, c\}$ and $C_{3}:\{c, a\}$ where $a, b, c$ are the object ids.

In the case of Elpis, however, the nodes submit to the ownership transition taking place in the system as they learn about it and thereby converge on the contention set. The nodes learn in two phases. (1) If a node $p_{1}$ receives a NACK message for a Prepare or a Commit message from $p_{2}$, that implies $p_{2}$ must have sent an $A C K$ to some node $p_{j}$ (could be $p_{2}$ itself). We piggyback this information $\left(\operatorname{tag}\left(p_{j}\right)\right.$, and epoch of ACK $\left.\left(p 2 \rightarrow p_{j}\right)\right)$ in $\operatorname{Defer}(p 2 \rightarrow p 1)$ (Algo 5 line 24, Algo 3 line 16) messages and let $p 1$ pick the best node to deferTo (Algo 6 line 13). Without collision recovery (CR), however, this can result in a case where the set $\left\{p_{1}, p_{2}, p_{3}\right\}$ defers to $\left\{p_{3}, p_{1}, p_{2}\right\}$. (2) Using CR, we force at least a quorum of nodes (Algo 6 line 26) to Trust the same node to have a chance to acquire the ownership. If the set now is $\left\{p_{1}, p_{2}, p_{3}\right\}$ defer to $\left\{p_{1}, p_{1}, p_{3}\right\}, p_{2}$ would not retry ownership acquisition for $a b$, but $p_{1}$ and $p_{3}$ would (contending on $c$ ) and either acquire the ownership by completing Prepare and Commit or follow (1) and (2) as above to eventually have a single owner. Additionally, Elpis guarantees liveness and consistency in the presence of Byzantine faults, whereas $\mathrm{M}^{2}$ Paxos does not.

As part of the Cross Fault Tolerance (XFT) [24], model the authors propose XPaxos which uses $2 t+1$ processes. The protocol is executed by a synchronous group of $t+1$ active processes with a fixed leader for the group. In the presence of faults, XPaxos transitions to a new group of $t+1$ processes using a view - change mechanism. XPaxos provides similar performance to Paxos while providing higher reliability by tolerating Byzantine faults and is optimized for the $t=1$ case but does not scale well with the number of faults.

Elpis uses Cross Fault Tolerance (XFT), the same system model as XPaxos but the leaderless protocol of Elpis with all $2 t+1$ active processes differs from XPaxos which uses fixed synchronous groups $(\mathrm{sg})$ of size $t+1$ with a fixed leader. XPaxos works by determining $\left(\begin{array}{c}n \\ t+1\end{array}\right) s g$ groups with active groups switching via a view-change mechanism in case of faults until a $s g$ with correct processes found. For higher $n$ and $t$, the number of such groups increases exponentially. However, in the worst case of Elpis, a client has to contact a maximum of $t+1$ processes.

\section{Conclusion}

This paper presented Elpis, the first multi-leader XFT protocol that overcomes the drawbacks of XPaxos, a single-leader protocol. Elpis implements generalized consensus. By assigning different and independent objects to different processes, such that the need for ordering is limited to local scope, each governed by one of the processes, and transfers ownership when needed. This way operations on disjoint collections of objects trivially commute, and Elpis can decide on such commands in just two communication delays while ownership transfer adds an additional delay. The efficacy of this approach is further validated by evaluation as it leads to significant performance gains over XPaxos and other BFT protocols. For the geo-replicated setting, Elpis achieves low latency for clients due to the ownership of objects accessed by the clients at the local process and high throughput due to the leaderless approach providing inherent load balancing. Hence, Elpis is an attractive option for building georeplicated fault-tolerant systems as not only does it provides better performance than BFT protocols but, it also offers higher reliability than CFT protocols.

\section{Acknowledgments}

We thank the anonymous reviewers for their valuable comments which have significantly improved the paper. This work is supported in part by NSF under grant CNS 1523558 and AFOSR under grant FA9550-15-1-0098. 


\section{References}

[1] 2012. AWS Service Event in the US-East Region: October 22, 2012. (2012). https://aws.amazon.com/message/680342/

[2] 2018. cockroach: CockroachDB - the open source, cloud-native SQL database. https://github.com/cockroachdb/cockroach original-date: 2014-02-06T00:18:47Z.

[3] 2018. etcd: Distributed reliable key-value store for the most critical data of a distributed system. https://github.com/coreos/etcd original-date: 2013-07-06T21:57:21Z.

[4] 2019. Google App Engine: 02 January 2019. (2019). https://status. cloud.google.com/incident/appengine/19001

[5] 2019. Google Compute Engine: November 05, 2018. (2019). https: //status.cloud.google.com/incident/compute/18012

[6] 2019. Home / YugaByte DB. https://www.yugabyte.com/. Accessed: 2019-09-04

[7] Balaji Arun, Sebastiano Peluso, Roberto Palmieri, Giuliano Losa, and Binoy Ravindran. 2017. Speeding up Consensus by Chasing Fast Decisions. arXiv:1704.03319 [cs] (April 2017). http://arxiv.org/abs/1704. 03319 arXiv: 1704.03319.

[8] Pierre-Louis Aublin, Rachid Guerraoui, Nikola Kneevi, Vivien Quéma, and Marko Vukoli. 2015. The Next 700 BFT Protocols. ACM Trans. Comput. Syst. 32, 4 (Jan. 2015), 12:1-12:45. https://doi.org/10.1145/ 2658994

[9] Bela Ban. 2002. JGroups, a toolkit for reliable multicast communication. (2002).

[10] Alysson Neves Bessani and Marcel Santos. 2011. Bft-smart-highperformance byzantine-faulttolerant state machine replication.

[11] Eric A Brewer. 2000. Towards robust distributed systems. In PODC, Vol. 7.

[12] Mike Burrows. 2006. The Chubby lock service for loosely-coupled distributed systems. In Proceedings of the 7th symposium on Operating systems design and implementation. USENIX Association, 335-350.

[13] Brad Calder, Ju Wang, Aaron Ogus, Niranjan Nilakantan, Arild Skjolsvold, Sam McKelvie, Yikang Xu, Shashwat Srivastav, Jiesheng Wu, Huseyin Simitci, et al. 2011. Windows Azure Storage: a highly available cloud storage service with strong consistency. In Proceedings of the Twenty-Third ACM Symposium on Operating Systems Principles. ACM, 143-157.

[14] Apache Cassandra. 2014. Apache cassandra. Website. Available online at http://planetcassandra. org/what-is-apache-cassandra (2014), 13.

[15] Miguel Castro and Barbara Liskov. 1999. Practical Byzantine fault tolerance. In OSDI, Vol. 99. 173-186.

[16] James C. Corbett, Jeffrey Dean, Michael Epstein, Andrew Fikes, Christopher Frost, J. J. Furman, Sanjay Ghemawat, Andrey Gubarev, Christopher Heiser, Peter Hochschild, Wilson Hsieh, Sebastian Kanthak, Eugene Kogan, Hongyi Li, Alexander Lloyd, Sergey Melnik, David Mwaura, David Nagle, Sean Quinlan, Rajesh Rao, Lindsay Rolig, Yasushi Saito, Michal Szymaniak, Christopher Taylor, Ruth Wang, and Dale Woodford. 2013. Spanner: Googles Globally Distributed Database. ACM Trans. Comput. Syst. 31, 3 (Aug. 2013), 8:1-8:22. https://doi.org/10.1145/2491245

[17] Patrick Hunt, Mahadev Konar, Flavio P Junqueira, and Benjamin Reed. 2010. ZooKeeper: Wait-free coordination for Internet-scale systems. (2010), 14.

[18] Ramakrishna Kotla, Lorenzo Alvisi, Mike Dahlin, Allen Clement, and Edmund Wong. 2007. Zyzzyva: speculative byzantine fault tolerance In ACM SIGOPS Operating Systems Review, Vol. 41. ACM, 45-58.

[19] Leslie Lamport. 2001. Paxos made simple. ACM Sigact News 32, 4 (2001), 18-25.

[20] Leslie Lamport. 2002. Specifying systems: the TLA+ language and tools for hardware and software engineers. Addison-Wesley Longman Publishing Co., Inc.

[21] Leslie Lamport. 2005. Generalized Consensus and Paxos. (2005), 63.
[22] Leslie Lamport. 2006. Fast Paxos. Distributed Computing 19 (Oct. 2006). https://www.microsoft.com/en-us/research/publication/fast-paxos/

[23] Leslie Lamport, Robert Shostak, and Marshall Pease. 1982. The Byzantine Generals Problem. ACM Transactions on Programming Languages and Systems 4, 3 (July 1982), 382-401. https://doi.org/10.1145/357172. 357176

[24] Shengyun Liu, Paolo Viotti, Christian Cachin, Vivien Quéma, and Marko Vukolic. 2016. XFT: Practical Fault Tolerance beyond Crashes.. In OSDI. 485-500.

[25] J-P Martin and Lorenzo Alvisi. 2006. Fast byzantine consensus. IEEE Transactions on Dependable and Secure Computing 3, 3 (2006), 202-215.

[26] Iulian Moraru, David G. Andersen, and Michael Kaminsky. 2013. There is more consensus in Egalitarian parliaments. ACM Press, 358-372. https://doi.org/10.1145/2517349.2517350

[27] Diego Ongaro and John K. Ousterhout. 2014. In search of an understandable consensus algorithm.. In USENIX Annual Technical Conference. 305-319.

[28] S. Peluso, A. Turcu, R. Palmieri, G. Losa, and B. Ravindran. 2016. Making Fast Consensus Generally Faster. In 2016 46th Annual IEEE/IFIP International Conference on Dependable Systems and Networks (DSN). 156-167. https://doi.org/10.1109/DSN.2016.23

[29] Fred B Schneider. 1993. Replication management using the statemachine approach, Distributed systems. (1993).

[30] Swaminathan Sivasubramanian. 2012. Amazon dynamoDB: a seamlessly scalable non-relational database service. In Proceedings of the 2012 ACM SIGMOD International Conference on Management of Data. ACM, 729-730.

[31] Mohammad Reza Khalifeh Soltanian and Iraj Sadegh Amiri. 2016. Chapter 1 - Introduction. In Theoretical and Experimental Methods for Defending Against DDOS Attacks, Mohammad Reza Khalifeh Soltanian and Iraj Sadegh Amiri (Eds.). Syngress, 1 - 5. https: //doi.org/10.1016/B978-0-12-805391-1.00001-8 